\title{
The PDEs and Numerical Scheme for Derivatives under Uncertainty Volatility
}

\author{
Yulian Fan \\ Department of mathematics, North China University of Technology, China \\ Correspondence should be addressed to Yulian Fan; fanyl@ncut.edu.cn
}

Received 27 August 2018; Accepted 14 May 2019; Published 29 May 2019

Academic Editor: Mariusz Michta

Copyright ( 2019 Yulian Fan. This is an open access article distributed under the Creative Commons Attribution License, which permits unrestricted use, distribution, and reproduction in any medium, provided the original work is properly cited.

\begin{abstract}
We use the stochastic differential equations (SDE) driven by G-Brownian motion to describe the basic assets (such as stocks) price processes with volatility uncertainty. We give the estimation method of the SDE's parameters. Then, by the nonlinear FeynmanKac formula, we get the partial differential equations satisfied by the derivatives. At last, we give a numerical scheme to solve the nonlinear partial differential equations.
\end{abstract}

\section{Introduction}

The financial asset pricing theory is usually under the framework of a stochastic model: a set of future scenarios $(\Omega, \mathscr{F})$ and a probability measure $P$ on these outcomes. However, there are many circumstances in financial decision making where the decision-maker or risk manager is not able to attribute a precise probability to future outcomes. This situation has been called "uncertainty" by [1], to contrast with "risk" when we are able to specify a unique probability measure on future outcomes. Then this kind of uncertainty is called Knight uncertainty, ambiguity, or model uncertainty. In his 1961 thesis, [2] established a distinction between aversion to risk, lack of knowledge of future outcomes, aversion to model uncertainty, and lack of knowledge of their probabilities and showed that aversion to model uncertainty can strongly affect decision-makers' behavior and resolve some paradoxes of classical decision theory, such as the equity premium puzzle (see [3]) and the home-bias puzzle. More recently, model uncertainty aversion has shown to have important consequences in macroeconomics $([4,5])$ and price behavior in capital markets $([6-8])$.

The worst case or "maxmin" approach has been proposed for evaluating uncertain outcomes in presence of model uncertainty. And it has been axiomatized by [9]. It proposed a system of axioms under which an agent facing model uncertainty chooses among a set A of feasible alternatives by maximizing a "robust" version of expected utility (also called "maxmin" expected utility), obtained by taking the worst case over all models:

$$
\max _{X \in A} \min _{P \in \mathscr{P}} E^{P}[u(X)] .
$$

The risk aversion of the decision-maker is captured by the utility function $u$, while the aversion to model uncertainty is captured by taking the infimum over all models in $\mathscr{P}$. The worst-case approach clearly distinguishes model uncertainty from risk: the latter is treated by averaging over scenarios with a given model while the former is treated by taking the infimum over models.

The worst case idea has resurfaced in the recent literature on sublinear expectation theory (see S. Peng's series papers) and risk measures $([10,11])$. It is proved that there exists a family of linear expectations $\left\{E_{\theta}\right\}_{\theta \in \Theta}$ such that a sublinear expectation has the following representation (see $[10,11])$ :

$$
\mathbb{E}[X]=\max _{\theta \in \Theta} E_{\theta}[X] .
$$

Reference [12] introduced $g$-expectation, and [6] proposed to use g-expectation for a robust valuation: $\mathbb{E}^{g}[X]=$ $\sup _{\theta \in \Theta} E_{\theta}[X]$, where each probability $P_{\theta}$ is absolutely continuous with respect to the "reference measure" $P_{0}$. The absolutely continuity with respect to the reference measure implies that the uncertainty only comes from the drift term and thus is called drift uncertainty. Reference [13] proved that, for any contingent claims $X$, the super evaluation 
of $X, \sup _{\theta \in \Theta} E_{\theta}[X]$, is a $g$-expectation. And under certain conditions, a consistent expectation dominated by a $g_{\mu^{-}}$ expectation can be expressed as a $g$-expectation. Therefore, in the situation of drift uncertainty, finding the generator $g$ is a key step for solving the value $\mathbb{E}^{g}\left[X \mid \mathscr{F}_{t}\right]$. Once $g$ is reconstructed, we can then apply a rapidly developed analysis and numerical results in the last decade to study and to calculate $\mathbb{E}^{g}\left[X \mid \mathscr{F}_{t}\right]$.

But when the uncertainty comes from the volatility coefficient, the probabilities $\left\{P_{\theta}\right\}_{\theta \in \Theta}$ are mutually singular. This type of uncertainty was initially studied by [14] for the superhedging of European type contingent claims whose payoffs depend only on the basic asset's terminal value. In this case the value of the derivatives should satisfy the BSB equations. Reference [15] studied the corresponding discrete-time case. For path-dependence option, [13] and [16] established theoretical frameworks respectively. Reference [13] defined a super evaluation operator which is monotonic, constant preserving, and filtration consistent and satisfies zero-one law. Then [17-19] introduced a sublinear expectation under which the increments of the canonical process $\left(B_{t}\right)_{t \geq 0}$ is zeromean, independent, stationary, and G-normally distributed. Under this case, the canonical process is called G-Brownian motion and the corresponding sublinear expectation is called G-expectation. The G-normal distribution random variables have no mean uncertainty but can have variance uncertainty. G-Brownian motion has a very rich and interesting new structure which nontrivially generalizes the classical Brownian motion. Under this framework, [20] numerically studied the pricing of Asian options when the volatility of the underlying asset is uncertain. Reference [16] proved that the value of any bounded contingent claim could be expressed as the supremum of its expectation over some set of martingale measure.

In this paper, we use the stochastic differential equations driven by G-Brownian motion to describe the stock price processes with volatility uncertainty. We give the estimation method of the parameters. Then by the nonlinear FeynmanKac formula, we get the partial differential equations satisfied by the derivatives. We also give the discretization scheme of the corresponding nonlinear partial differential equations. This procedure can be used to pricing derivatives with some kind of path-dependent payoffs and it is practically applicable.

The paper is organized as follows. In Section 2, we use stochastic differential equations driven by G-Brownian motion to describe stock price processes with volatility uncertainty and give the estimation method of the parameters. Section 3 introduces the nonlinear Feynman-Kac formula to price derivatives based on the above stocks. Section 4 gives the discretization scheme of the corresponding nonlinear partial differential equations.

\section{The Stock Price Process and the Estimation of the Parameters}

One reality example of model uncertainty is the nonuniqueness of the pricing measures or martingale measures in incomplete markets. While the existence of the so-called "volatility risk" in option trading is a concrete manifestation of market incompleteness. Volatility risk comes from the uncertainty of the volatility. This type of uncertainty was initially studied by [14]. These papers addressed the issue of the derivative pricing and hedging in an uncertain volatility environment and propose that the market operated under the assumption that the volatility of the price is restricted in a bounded set, without other information; i.e., the price paths of the basic asset such as stock are the following Itô processes:

$$
d S_{t}^{Z}=\mathrm{S}_{t}^{Z}\left(\sigma_{t}^{Z} d Z_{t}+\mu_{t}^{Z} d t\right), \quad S_{0}^{Z}=y,
$$

where $\sigma_{t}^{Z}, \mu_{t}^{Z}$ are nonanticipative functions with $\underline{\sigma}^{Z} \leq \sigma_{t}^{Z} \leq$ $\bar{\sigma}^{Z}$, and $Z$ is the classical Brownian motion. $\underline{\sigma}^{Z}, \bar{\sigma}^{Z}$ are constants representing the upper and lower bounds of the volatility.

Motivated by the problem of coherent risk measures under the volatility uncertainty, $[17,19]$ introduced a sublinear expectation on a well-defined space $L_{G}^{1}(\Omega)$ under which the increments of the canonical process $\left(B_{t}\right)_{t \geq 0}$ are zeromean, independent, and stationary and can be proved to be G-normally distributed (see [18]). As we have mentioned in previous sections, this type of processes is G-Brownian motion and the corresponding sublinear expectation $\widehat{\mathbb{E}}[\cdot]$ is G-expectation. The G-Brownian motion $B_{t}$ is $N(\{0\} \times t \Sigma)$ distributed. $t \Sigma$ characterizes the variance uncertainty of $B_{t}$.

In this paper, we will use the stochastic differential equation driven by the G-Brownian motion, instead of (3), to characterize the basic asset price process. The volatility uncertainty will be embodied in the variance uncertainty of the G-Brownian motion $B$.

For simplicity, we assume that $d=1$ and $n=1$.

For the G-Brownian motion, there are two important parameters $\underline{\sigma}$ and $\bar{\sigma}$. So the first important thing is to estimate $\underline{\sigma}$ and $\bar{\sigma}$ using the market data.

Let us assume there is a referenced asset or portfolio in the market. This is true in the real market, for example, the Dow Jones index in America stock markets, the UK FTSE in Europe stock markets, and the Nikkei 225 in Asia stock markets. We assume the price process of the reference asset follows the stochastic differential equation:

$$
d A_{t}=\mu A_{t} d t+A_{t} d B_{t}, \quad A_{0}=a_{0}, t \in[0, T] .
$$

where $\mu$ is the expected growth rate of the economy in the market and can be estimated using economic data, so we can take $\mu$ as known constant. If there is no arbitrage, $\mu$ can be replaced by the riskless interest rate (see [14] or [21]).

Note we let the coefficient before the term $A_{t} d B_{t}$ to be 1 . This is because if $B$ is a G-Brownian motion with uncertain variance $\sigma^{2} \in\left[\underline{\sigma}^{2}, \bar{\sigma}^{2}\right]$, then, for any constant $\lambda, \lambda B$ still is a G-Brownian motion but with uncertain variance $\sigma_{\lambda}^{2} \epsilon$ $\left[\lambda^{2} \underline{\sigma}^{2}, \lambda^{2} \bar{\sigma}^{2}\right]$.

From (4), we have

$$
\ln A_{t}-\ln a_{0}=\mu t-\frac{1}{2}\langle B\rangle_{t}+B_{t},
$$


and

$$
\begin{aligned}
\mathbb{E}\left[\ln A_{t}-\ln a_{0}\right] & =\mu t-\frac{1}{2} \underline{\sigma}^{2} t, \\
-\mathbb{E}\left[-\left(\ln A_{t}-\ln a_{0}\right)\right] & =\mu t-\frac{1}{2} \bar{\sigma}^{2} t
\end{aligned}
$$

Since $\mu$ is known constant, the parameters should be estimated as $\underline{\sigma}^{2}$ and $\bar{\sigma}^{2}$. We use historical data to estimate them. Let $A_{i}$ to be the referenced asset price at the end of time $i, i=0,1, \ldots, n$, and $t_{i+1}-t_{i}=\Delta t$. Let $C_{i}=\ln A_{i}-\ln A_{i-1}-$ $\mu \Delta t, i=1,2, \ldots, n$; then

$$
\begin{aligned}
C_{i}=-\frac{1}{2}\left(\langle B\rangle_{t_{i}}-\langle B\rangle_{t_{i-1}}\right)+\left(B_{t_{i}}-B_{t_{i-1}}\right), & \\
& i=1,2, \ldots, n .
\end{aligned}
$$

Since $\langle B\rangle$ and $B$ are processes with independent and identically distributed increments under $\widehat{\mathbb{E}}, C_{i+1} \stackrel{\mathrm{d}}{=} C_{i}, i=$ $1,2, \ldots, n$ and $C_{i+1}$ is independent from $C_{i}, i=1,2, \ldots, n$. Then from the new central limit theorem of [22], we know that $(1 / n) \sum_{i=1}^{n} C_{i}$ converges in law to the worst case distribution $N\left(\left[-(1 / 2) \bar{\sigma}^{2} \Delta t,-(1 / 2) \underline{\sigma}^{2} \Delta t\right] \times 0\right)$, with $-(1 / 2) \underline{\sigma}^{2} \Delta t=$ $\widehat{\mathbb{E}}\left[C_{i}\right]$ and $-(1 / 2) \bar{\sigma}^{2} \Delta t=-\widehat{\mathbb{E}}\left[-C_{i}\right]$. It means when $n \longrightarrow$ $\infty$, the number $(1 / n) \sum_{i=1}^{n} C_{i}$ can take any value inside $\left[-(1 / 2) \bar{\sigma}^{2} \Delta t,-(1 / 2) \underline{\sigma}^{2} \Delta t\right]$. Thus we can calculate $\hat{\mathbb{E}}\left[C_{i}\right]$ by

$$
\limsup _{n \rightarrow \infty} \frac{1}{n} \sum_{i=1}^{n} C_{i}=\widehat{\mathbb{E}}\left[C_{i}\right]=-\frac{1}{2} \underline{\sigma}^{2} \Delta t
$$

This is the new nonlinear Monte-Carlo approach provided by [22]. Therefore, using historical data we can estimate the value of $\underline{\sigma}^{2}$ and $\bar{\sigma}^{2}$.

Then for any asset in the market, we assume its price process follows the stochastic differential equation:

$$
d S_{t}=\alpha S_{t} d t+\gamma S_{t} d B_{t}, \quad S_{0}=s_{0}, t \in[0, T],
$$

where $\alpha, \gamma$ are assumed to be constants.

Then

$$
S_{t}=S_{0} \exp \left(\alpha t-\frac{1}{2} \gamma^{2}\langle B\rangle_{t}+\gamma B_{t}\right)
$$

and

$$
\begin{aligned}
\mathbb{E}\left[\ln S_{t}-\ln S_{0}\right] & =\alpha t-\frac{1}{2} \gamma^{2} \underline{\sigma}^{2} t, \\
-\mathbb{E}\left[-\left(\ln S_{t}-\ln S_{0}\right)\right] & =\alpha t-\frac{1}{2} \gamma^{2} \bar{\sigma}^{2} t
\end{aligned}
$$

Using the above nonlinear Monte-Carlo approach, we can get the estimations of $\mathbb{E}\left[\ln S_{t}-\ln S_{t-\Delta t}\right]$ and $-\mathbb{E}\left[-\left(\ln S_{t}-\right.\right.$ $\left.\ln S_{t-\Delta t}\right)$ ]. Solve (11), we can get $\alpha$ and $\gamma$. The parameters $\alpha, \gamma$ determine the coefficients of the partial differential equations satisfied by the derivatives based on this asset.

\section{The Nonlinear Feynman-Kac Formula}

As we stated previously, in incompleteness markets the pricing measures or martingale measures are not unique which lead to the uncertainty of the model. One reality example of model uncertainty is the volatility uncertainty. This type of uncertainty was initially studied by [14]. They assume the price paths of the basic assets such as stocks follow the Itô processes (3):

$$
d S_{s}^{Z}=S_{s}^{Z}\left(\sigma_{s}^{Z} d Z_{s}+\mu_{s}^{Z} d s\right), \quad S_{t}^{Z}=y,
$$

where $\sigma_{t}^{Z}, \mu_{t}^{Z}$ are nonanticipative functions with $\underline{\sigma}^{Z} \leq \sigma_{t}^{Z} \leq$ $\bar{\sigma}^{Z}$, and $Z$ is the classical Brownian motion.

For simplicity, we assume $\sigma_{t}^{Z}, \mu_{t}^{Z}$ are constants denoted as $\sigma^{Z}, \mu^{Z}$. Then

$$
\frac{d S_{s}^{Z}}{S_{s}^{Z}}=\sigma^{Z} d Z_{s}+\mu^{Z} d s, \quad S_{t}^{Z}=y,
$$

For every admissible pricing measure $P \in \mathscr{P}$, the stock price's expected rate of return is always $\mu^{Z}$, while the stock price volatility lies in interval $\underline{\sigma}^{Z}, \bar{\sigma}^{Z}$.

In this section, we will use stochastic differential equations driven by G-Brownian motion as the basic assets price processes. We will find that the stochastic differential equations driven by G-Brownian motion include the uncertain volatility model as a special case.

We assume that $d=1, n=1$, and the stock price process follows the stochastic differential equation:

$$
d X_{s}^{t, x}=\alpha X_{s}^{t, x} d s+\gamma X_{s}^{t, x} d B_{s}, \quad X_{t}^{t, x}=x, s \in[t, T],
$$

where $\left(B_{s}\right)_{s \geq 0}$ is a G-Brownian motion with $B_{s} \sim N(\{0\} \times$ $\left.\left[\underline{\sigma}^{2} s, \bar{\sigma}^{2} s\right]\right)$ and $\alpha, \gamma$ are given constant.

Reference (14) is equivalent to

$$
\frac{d X_{s}^{t, x}}{X_{s}^{t, x}}=\alpha d s+\gamma d B_{s}, \quad X_{t}^{t, x}=x, s \in[t, T],
$$

The G-expected rate of return of the stock price (14) is $\alpha$, while the stock price volatility lies in interval $[|\gamma| \underline{\sigma},|\gamma| \bar{\sigma}]$. If we let $\alpha=\mu^{Z},|\gamma| \underline{\sigma}=\underline{\sigma}^{Z},|\gamma| \bar{\sigma}=\bar{\sigma}^{Z}$, then the stock prices (3) and (14) have the same expected rate of return and volatility.

Let us consider a financial contract based on stock price process (14) with terminal payoff $\Phi\left(X_{T}^{x}\right)$ and cash-flow $f\left(X_{t}^{x}\right)$ at time $t$ during holding period $[0, T]$. We assume $\Phi(\cdot)$ and $f(\cdot)$ are Lipschitz functions.

In financial markets with model uncertainty, the worst case approach is commonly used in asset pricing such as $[14,23,24]$ and risk measure such as $[10,11]$. The sublinear expectation embodies the idea of worst case. If the buyer or seller of the contract pricing is based on the worst case hedging, the value of this contract will be

the ask price: $\mathbb{E}\left[\Phi\left(X_{T}^{x}\right)+\int_{t}^{T} f\left(X_{s}^{x}\right) d s\right]$, the bid price: $-\mathbb{E}\left[-\Phi\left(X_{T}^{x}\right)-\int_{t}^{T} f\left(X_{s}^{x}\right) d s\right]$.

We just consider the ask price. The bid price is similar. Define

$$
u(t, x)=\mathbb{E}\left[\Phi\left(X_{T}^{x}\right)+\int_{t}^{T} f\left(X_{s}^{x}\right) d s\right]
$$


By the nonlinear Feynman-Kac formula of [25], $u(t, x)$ is a viscosity solution of the following PDE:

$$
\begin{aligned}
& \partial_{t} u+\frac{1}{2} \sup _{\underline{\sigma}^{2} \leq \sigma^{2} \leq \bar{\sigma}^{2}}\left(D^{2} u \gamma^{2} x^{2} \cdot \sigma^{2}\right)+D u \cdot \alpha x+f(x) \\
& \quad=0 \\
& u(T, x)=\Phi(x) .
\end{aligned}
$$

Taking the riskless interest rate into account, the ask price of the contract will be $\mathbb{E}\left[e^{-r T} \Phi\left(X_{T}^{x}\right)+\int_{t}^{T} e^{-r s} f\left(X_{s}^{x}\right) d s\right]$. Define

$$
u(t, x)=\left[e^{-r T} \Phi\left(X_{T}^{x}\right)+\int_{t}^{T} e^{-r s} f\left(X_{s}^{x}\right) d s\right]
$$

Correspondingly, $u(t, x)$ satisfies the following PDE:

$$
\begin{aligned}
& \partial_{t} u+\frac{1}{2} \sup _{\underline{\sigma}^{2} \leq \sigma^{2} \leq \bar{\sigma}^{2}}\left(D^{2} u \gamma^{2} x^{2} \cdot \sigma^{2}\right)+D u \cdot \alpha x-r u \\
& \quad+f(x)=0, \\
& u(T, x)=\Phi(x) .
\end{aligned}
$$

PDE (17) and (19) is a generalization of the BSB equations used in [14], since we take some kind of path-dependent derivatives into consideration.

\section{Solving the PDE}

Let $v(\tau, x)=u(T-\tau, x)$; then $\operatorname{PDE}(17)$ can be written as

$$
\begin{aligned}
& -\partial_{\tau} v+\frac{1}{2} \sup _{\underline{\sigma}^{2} \leq \sigma^{2} \leq \bar{\sigma}^{2}}\left(D^{2} v \gamma^{2} x^{2} \cdot \sigma^{2}\right)+D v \cdot \alpha x+f(x) \\
& =0, \\
& v(0, x)=\Phi(x) .
\end{aligned}
$$

Before proceeding to the discretization scheme, we first consider the boundary conditions of (20).

When $\tau=0$, we already have $v(0, x)=\Phi(x)$ which is the specified contract payoff at the expiration. If $x=0$, then we have $X_{s}^{t, 0} \equiv 0$, and the equation is reduced to $\partial_{\tau} v=f(0)$. As $x \longrightarrow+\infty$, we have the Dirichlet condition

$$
v(\tau, x)=\Psi(\tau, x),
$$

where $\Psi(\tau, x)$ can be determined by financial reasoning. For computational purpose, we truncate the asset region $(0,+\infty)$ into $\left(0, x_{\max }\right)$, where $x_{\max }$ is sufficiently large to ensure the accuracy of the solution (ref [26]). Then (21) becomes

$$
v\left(\tau, x_{\max }\right)=\Psi\left(\tau, x_{\max }\right) .
$$

Now, let us consider the discretization of (20). Denote the solution domain $D=D_{\text {in }} \cup D_{b}$, where $D_{\text {in }}$ is the interior points and $D_{b}$ is the boundary points. Let $\bar{x}=(x, \tau), v(\bar{x})=$ $v(\tau, x), D^{2} v=v_{x x}, D v=\left(v_{x}, v_{\tau}\right)$.
Then we can write the general form of the PDE in a way that includes boundary conditions

$$
\begin{aligned}
& g\left(D^{2} v(\bar{x}), D v(\bar{x}), v(\bar{x}), \bar{x}\right) \\
& \quad=g_{i n}\left(D^{2} v(\bar{x}), D v(\bar{x}), v(\bar{x}), \bar{x}\right), \quad \bar{x} \in D_{i n} \\
& g\left(D^{2} v(\bar{x}), D v(\bar{x}), v(\bar{x}), \bar{x}\right) \\
& \quad=g_{b}\left(D^{2} v(\bar{x}), D v(\bar{x}), v(\bar{x}), \bar{x}\right), \quad \bar{x} \in D_{b},
\end{aligned}
$$

where

$$
\begin{aligned}
g_{\text {in }} & \left(D^{2} v(\bar{x}), D v(\bar{x}), v(\bar{x}), \bar{x}\right) \\
= & v_{\tau}-\frac{1}{2} \sup _{\underline{\sigma}^{2} \leq \sigma^{2} \leq \bar{\sigma}^{2}}\left(D^{2} v \gamma^{2} x^{2} \cdot \sigma^{2}\right)-D v \cdot \alpha x \\
& \quad-f(x)=0 \\
g_{b}( & \left.D^{2} v(\bar{x}), D v(\bar{x}), v(\bar{x}), \bar{x}\right) \\
= & \text { specified boundary conditions. }
\end{aligned}
$$

Define a grid $0=x_{0}<x_{1}<\ldots<x_{M}=x_{\max }$ and a set of timesteps $0=\tau_{0}<\tau_{1}<\ldots<\tau_{N}=T$. Let the discretization parameter $h$ be given by

$$
\begin{gathered}
\max _{i \in\{0, \ldots, M-1\}}\left(x_{i+1}-x_{i}\right)=C_{1} h \\
\max _{n \in\{0, \ldots, N-1\}}\left(\tau^{n+1}-\tau^{n}\right)=C_{2} h,
\end{gathered}
$$

with $C_{1}, C_{2}=$ constant. To be simplified, we assume the discretization is even in space and time; i.e., $x_{i+1}-x_{i}=\triangle x, i=$ $0, \ldots, M-1 ; \tau^{n+1}-\tau^{n}=\Delta t, n=0, \ldots, N-1$

Let $v_{i}^{n}$ be the approximate value of the solution; i.e., $v_{i}^{n} \simeq v\left(\tau^{n}, x_{i}\right)$. Denote the discretization of $g\left(D^{2} v(\bar{x}), D v(\bar{x})\right.$, $v(\bar{x}), \bar{x})$ at node $\bar{x}_{i}^{n+1}=\left(\tau^{n+1}, x_{i}\right)$ as $G_{i}^{n+1}:=G_{i}^{n+1}\left(\triangle \bar{x}, v_{i}^{n+1}\right.$, $\left.v_{i+1}^{n+1}, v_{i-1}^{n+1}, v_{i}^{n}\right)$ with

$$
\begin{aligned}
G_{i}^{n+1}= & \frac{v_{i}^{n+1}-v_{i}^{n}}{\Delta t} \\
& -\frac{1}{2} \sup _{\underline{\sigma} \leq \sigma^{n+1} \leq \bar{\sigma}} \frac{v_{i+1}^{n+1}-2 v_{i}^{n+1}+v_{i-1}^{n+1}}{\triangle x} \gamma^{2} x_{i}^{2}\left(\sigma^{n+1}\right)^{2} \\
& -\frac{v_{i+1}^{n+1}-v_{i}^{n+1}}{\triangle x} \alpha x_{i}-f\left(x_{i}\right), \\
G_{0}^{n+1}= & v_{0}^{n+1}-v_{0} ; \\
G_{M}^{n+1}= & v_{M}^{n+1}-\Psi\left(\tau^{n+1}, x_{\max }\right) ; \\
G_{i}^{1}= & v_{i}^{1}-\Phi\left(x_{i}\right) ;
\end{aligned}
$$

where $v_{0}$ is the solution of equation $\partial_{t} v=f(0)$, with boundary condition $v(0,0)=\Phi(0)$. 
$G_{i}^{n+1}, i \neq 0, M ; n \neq 0, N$ can be written as

$$
\begin{gathered}
G_{i}^{n+1}=\frac{v_{i}^{n+1}-v_{i}^{n}}{\Delta t}-\frac{1}{2} \sup _{\underline{\sigma \leq \sigma^{n+1} \leq \bar{\sigma}}}\left\{a_{i}^{n+1}\left(\sigma^{n+1}\right) v_{i-1}^{n+1}\right. \\
\left.+c_{i}^{n+1}\left(\sigma^{n+1}\right) v_{i}^{n+1}+b_{i}^{n+1}\left(\sigma^{n+1}\right) v_{i+1}^{n+1}\right\}-f_{i},
\end{gathered}
$$

or

$$
\begin{aligned}
& \left(1-\frac{1}{2} \Delta t c_{i}^{n+1}\left(\widehat{\sigma}^{n+1}\right)\right) v_{i}^{n+1}-\frac{1}{2} \Delta t a_{i}^{n+1}\left(\widehat{\sigma}^{n+1}\right) v_{i-1}^{n+1} \\
& -\frac{1}{2} \Delta t b_{i}^{n+1}\left(\widehat{\sigma}^{n+1}\right) v_{i+1}^{n+1}=v_{i}^{n}+\Delta t f_{i} \\
& \widehat{\sigma}^{n+1}=\underset{\underline{\sigma} \leq \sigma^{n+1} \leq \bar{\sigma}}{\arg \max }\left(a_{i}^{n+1}\left(\sigma^{n+1}\right) v_{i-1}^{n+1}+c_{i}^{n+1}\left(\sigma^{n+1}\right) v_{i}^{n+1}\right. \\
& \left.+b_{i}^{n+1}\left(\sigma^{n+1}\right) v_{i+1}^{n+1}\right),
\end{aligned}
$$

where

$$
\begin{aligned}
a_{i}^{n+1}\left(\sigma^{n+1}\right) & =\frac{\gamma^{2} x_{i}^{2} \sigma^{2}}{\triangle x^{2}}, \\
b_{i}^{n+1}\left(\sigma^{n+1}\right) & =\frac{\gamma^{2} x_{i}^{2} \sigma^{2}}{\triangle x^{2}}+\frac{\alpha x_{i}}{\triangle x}, \\
c_{i}^{n+1}\left(\sigma^{n+1}\right) & =-\frac{2 \gamma^{2} x_{i}^{2} \sigma^{2}}{\triangle x^{2}}-\frac{\alpha x_{i}}{\triangle x}, \\
f_{i} & =f\left(x_{i}\right) .
\end{aligned}
$$

Let

$$
\begin{aligned}
v^{n} & =\left[v_{1}^{n}, \ldots, v_{M-1}^{n}\right]^{T}, \\
\sigma^{n} & =\left[\sigma_{1}^{n}, \ldots, \sigma_{M-1}^{n}\right]^{T}, \\
R^{n} & =\left[a_{1}^{n+1} v_{0}^{n+1}, 0, \ldots, 0, b_{N-1}^{n+1} v_{M}^{n+1}\right]_{M-1}^{T}, \\
A^{n+1} & =A^{n+1}\left(\sigma^{n+1}\right) \\
& =\left[\begin{array}{llll}
\frac{1}{2} c_{1}^{n+1} & \frac{1}{2} b_{1}^{n+1} & \\
\frac{1}{2} a_{2}^{n+1} & \frac{1}{2} c_{2}^{n+1} & \frac{1}{2} b_{2}^{n+1} & \\
\ddots & \ddots & \ddots \\
& & \frac{1}{2} a_{M-1}^{n+1} & \frac{1}{2} c_{M-1}^{n+1}
\end{array}\right]_{(M-1) \times(M-1)}
\end{aligned} .
$$

Then (28) can be written as the following matrix form:

$$
\begin{aligned}
{\left[I-\triangle t A^{n+1}\right] v^{n+1} } & =v^{n}+\Delta t R^{n}+\Delta t f_{i}, \\
\hat{\sigma}^{n+1} & =\underset{\underline{\sigma} \leq \sigma^{n+1} \leq \bar{\sigma}}{\arg \max }\left\{\left[A^{n+1} v^{n+1}+R^{n+1}\right]\right\} .
\end{aligned}
$$

If $\alpha>0$, then $a_{i}^{n+1}>0, b_{i}^{n+1}>0, c_{i}^{n+1}<0, i=1, \ldots, M$, $n=0, \ldots, N-1$. It is clear that $-A^{n+1}$ and $I-\triangle t A^{n+1}$ both are $M$-matrix. From the property of M-matrix, the solution of (31) exists and is unique.
On the other hand, if $\alpha<0$, we change the forward differencing term

$$
\frac{v_{i+1}^{n+1}-v_{i}^{n+1}}{\triangle x} \alpha x_{i}
$$

in the first discretization equation of (26) to backward differencing

$$
\frac{v_{i}^{n+1}-v_{i-1}^{n+1}}{\Delta x} \alpha x_{i} .
$$

Then $G_{i}^{n+1}, i \neq 0, M ; n \neq 0, N$ can be written as

$$
\begin{gathered}
G_{i}^{n+1}=\frac{v_{i}^{n+1}-v_{i}^{n}}{\Delta t}-\frac{1}{2} \sup _{\underline{\sigma} \leq \sigma^{n+1} \leq \bar{\sigma}}\left\{\bar{a}_{i}^{n+1}\left(\sigma^{n+1}\right) v_{i-1}^{n+1}\right. \\
\left.+\bar{c}_{i}^{n+1}\left(\sigma^{n+1}\right) v_{i}^{n+1}+\bar{b}_{i}^{n+1}\left(\sigma^{n+1}\right) v_{i+1}^{n+1}\right\}-f_{i},
\end{gathered}
$$

where $\bar{a}_{i}^{n+1}\left(\sigma^{n+1}\right)=\gamma^{2} x_{i}^{2} \sigma^{2} / \triangle x^{2}-\alpha x_{i} / \triangle x, \bar{b}_{i}^{n+1}\left(\sigma^{n+1}\right)=$ $\gamma^{2} x_{i}^{2} \sigma^{2} / \triangle x^{2}, \bar{c}_{i}^{n+1}\left(\sigma^{n+1}\right)=-2 \gamma^{2} x_{i}^{2} \sigma^{2} / \triangle x^{2}+\alpha x_{i} / \triangle x$. We also have $\bar{a}_{i}^{n+1}>0, \bar{b}_{i}^{n+1}>0, \bar{c}_{i}^{n+1}<0, i=1, \ldots, M, n=0, \ldots, N-$ 1. The matrix form of the discretization equation is similar to (31) and has unique solution.

It is important to ensure that we generate a numerical solution which is guaranteed to converge to the viscosity solution. It has been shown that HJB equation (20) satisfies the strong comparison property [27]. Therefore, there exists a unique continuous viscosity solution. Then, from [28], a numerical scheme converges to the viscosity solution if the method is consistent, stable, and monotone. Thus, we will show our numerical scheme satisfies these conditions.

Lemma 1 (stability). The discretization (26) is stable.

Proof. It follows from (28) that

$$
\begin{aligned}
(1 & \left.-\frac{1}{2} \Delta t c_{i}^{n+1}\right)\left|v_{i}^{n+1}\right| \\
\leq & \left|v_{i}^{n}\right|+\Delta t\left|f_{i}\right|+\frac{1}{2} \Delta t a_{i}^{n+1}\left|v_{i-1}^{n+1}\right|+\frac{1}{2} \Delta t b_{i}^{n+1}\left|v_{i+1}^{n+1}\right| \\
\leq & \left\|v^{n}\right\|_{\infty}+\Delta t\|f\|_{\infty} \\
& +\frac{1}{2} \triangle t\left(a_{i}^{n+1}+b_{i}^{n+1}\right)\left\|v^{n+1}\right\|_{\infty} .
\end{aligned}
$$

If $\left\|v^{n+1}\right\|_{\infty}=\left|v_{j}^{n+1}\right|, 0<j<M$, then when $\Delta t \longrightarrow 0$, we have

$$
\begin{aligned}
\left\|v^{n+1}\right\|_{\infty} & \leq \frac{\left\|v^{n}\right\|_{\infty}+\Delta t\|f\|_{\infty}}{1-(1 / 2) \Delta t\left(a_{i}^{n+1}+b_{i}^{n+1}+c_{i}^{n+1}\right)} \\
& =\frac{\left\|v^{n}\right\|_{\infty}+\Delta t\|f\|_{\infty}}{1-(1 / 2) \triangle t} \longrightarrow \\
\left\|v^{n}\right\|_{\infty} & \leq\left\|v^{0}\right\|_{\infty} .
\end{aligned}
$$

Otherwise, $\left\|v^{n+1}\right\|_{\infty}=\left|v_{0}^{n+1}\right|$ or $\left\|v^{n+1}\right\|_{\infty}=\left|v_{M}^{n+1}\right|$. Therefore,

$$
\left\|v^{n+1}\right\|_{\infty} \leq \max \left(\left\|v^{0}\right\|_{\infty}, \max _{0 \leq n \leq N}\left|v_{0}^{n}\right|, \max _{0 \leq n \leq N}\left|v_{N}^{n}\right|\right)
$$

with $v_{0}^{n}, v_{N}^{n}$ being the given Dirichlet boundary conditions. 
Lemma 2 (monotonicity). The discretization (26) is monotonic.

Proof. The lemma is trivially true on the boundary, so let us just see the case $0<i<M$ and $0<n \leq N$. For any $\epsilon>0$,

$$
\begin{aligned}
G_{i}^{n+1} & \left(\triangle \bar{x}, v_{i}^{n+1}, v_{i+1}^{n+1}+\epsilon, v_{i-1}^{n+1}, v_{i}^{n}\right) \\
- & G_{i}^{n+1}\left(\triangle \bar{x}, v_{i}^{n+1}, v_{i+1}^{n+1}, v_{i-1}^{n+1}, v_{i}^{n}\right)=\frac{1}{2} \\
\cdot & \sup _{\sigma}\left(\sigma^{n+1} \leq \bar{\sigma}\right. \\
+ & b_{i}^{n+1}\left(\sigma^{n+1}\right) v_{i-1}^{n+1}+c_{i}^{n+1}\left(\sigma^{n+1}\right) v_{i}^{n+1} \\
& +c_{i}^{n+1}\left(\sigma_{i+1}^{n+1}\right)-\frac{1}{2} \sup _{\underline{\sigma} \leq \sigma^{n+1} \leq \bar{\sigma}}\left(a_{i}^{n+1}\left(\sigma^{n+1}\right) v_{i-1}^{n+1}+b_{i}^{n+1}\left(\sigma^{n+1}\right)\left(v_{i+1}^{n+1}+\epsilon\right)\right) \leq \frac{1}{2} \\
& \cdot \sup _{\sigma}\left(-b_{i}^{n+1} \leq \bar{\sigma}\right.
\end{aligned}
$$

Since $b_{i}^{n+1}\left(\sigma^{n+1}\right)=\gamma^{2} x_{i}^{2} \sigma^{2} / \triangle x^{2}+\alpha x_{i} / \triangle x>0$ and $\sup _{\underline{\sigma \leq \sigma \leq \bar{\sigma}}} G_{1}(\sigma)-\sup _{\underline{\sigma \leq \sigma \leq \bar{\sigma}}} G_{2}(\sigma) \leq \sup _{\underline{\sigma \leq \sigma \leq \bar{\sigma}}}\left(G_{1}(\sigma)-G_{2}(\sigma)\right)$, similarly,

$$
\begin{aligned}
& G_{i}^{n+1}\left(\triangle \bar{x}, v_{i}^{n+1}, v_{i+1}^{n+1}, v_{i-1}^{n+1}+\epsilon, v_{i}^{n}\right) \\
& \leq G_{i}^{n+1}\left(\triangle \bar{x}, v_{i}^{n+1}, v_{i+1}^{n+1}, v_{i-1}^{n+1}, v_{i}^{n}\right), \\
& G_{i}^{n+1}\left(\triangle \bar{x}, v_{i}^{n+1}, v_{i+1}^{n+1}, v_{i-1}^{n+1}, v_{i}^{n}+\epsilon\right) \\
& \quad \leq G_{i}^{n+1}\left(\triangle \bar{x}, v_{i}^{n+1}, v_{i+1}^{n+1}, v_{i-1}^{n+1}, v_{i}^{n}\right) .
\end{aligned}
$$

Lemma 3 (consistency). The discretization (26) is consistent.

Proof. $g_{\text {in }}$ is continuous, so, at interior points, the upper semicontinuous envelope $g^{*}$ and the lower semicontinuous envelope $g_{*}$ are all equal to $g_{i n}$; i.e., $g^{*}=g_{*}=g_{i n}$. For all smooth bounded test functions $\phi(x)$,

$$
\begin{aligned}
& \lim \sup _{\substack{\triangle \bar{x} \longrightarrow 0 \\
\bar{x}_{i}^{n+1} \longrightarrow \bar{x} \\
\xi \longrightarrow 0}} G_{i}^{n+1}\left(\triangle \bar{x}, \phi\left(\bar{x}_{i}^{n+1}\right)+\xi, \phi\left(\bar{x}_{i+1}^{n+1}\right)+\xi,\right. \\
& \left.\phi\left(\bar{x}_{i-1}^{n+1}\right)+\xi, \phi\left(\bar{x}_{i}^{n}\right)\right) \\
& =\lim \sup _{\substack{\triangle \bar{x} \longrightarrow 0 \\
\bar{x}_{i}^{n+1} \longrightarrow \bar{x}}} g_{i n}\left(D^{2} \phi\left(\bar{x}_{i}^{n+1}\right), D \phi\left(\bar{x}_{i}^{n+1}\right), \phi\left(\bar{x}_{i}^{n+1}\right),\right. \\
& \left.\bar{x}_{i}^{n+1}\right) \underset{\substack{\xi \\
+}}{+O(\triangle \bar{x})+O(\xi)=g_{i n}=g^{*}=g_{*} .}
\end{aligned}
$$

The consistency is also satisfied on the boundary.

Therefore, we have the following.
Theorem 4. The solution of the discretization scheme (26) converges uniformly to the unique viscosity solution of PDE (20).

For PDE (19), using fully implicit timestepping and choosing forward, backward, or central differencing properly, we also can get the discretization equation converging uniformly to their unique viscosity solution.

\section{Conclusion}

In recent years, various case studies have indicated the importance of model uncertainty in financial market, even in macroeconomics, and some spectacular failures in risk management of derivatives have emphasized the consequences of neglecting model uncertainty.

Motivated by the problem of coherent risk measures under the volatility uncertainty, $[17,19]$ introduced GBrownian motion. The increments of G-Brownian motion are zero-mean, independent, and stationary and can be proved to be "G-normally distributed". Although the GBrownian motion in the sublinear expectation space has many properties just like the classical Brownian motion in linear expectation space, the increment of G-Brownian motion has uncertain variance and the quadratic variation process of G-Brownian motion is not a deterministic process unless $\underline{\sigma}=\bar{\sigma}$. In this paper, we give the estimation approach of the parameters of the stochastic differential equations driven by G-Brownian motion. The parameters determine the coefficients of the partial differential equations satisfied by the derivatives. Then we give a discretization scheme to solve the PDE. This procedure can be used to price derivatives based on basic asset with volatility uncertainty.

\section{Data Availability}

No data were used to support this study.

\section{Conflicts of Interest}

The author declares that there are no conflicts of interest regarding the publication of this article.

\section{References}

[1] F. Knight, Risk, Uncertainty and Profit, Houghton Mifflin, Boston, Mass, USA, 1921.

[2] D. Ellsberg, "Risk," Ambiguity, and the Savage Axioms, vol. 75, pp. 643-669, 1961.

[3] R. Mehra and E. C. Prescott, "The equity premium: a puzzle," Journal of Monetary Economics, vol. 15, no. 2, pp. 145-161, 1985.

[4] L. P. Hansen, T. J. Sargent, and T. D. Tallarini Jr., "Robust permanent income and pricing," Review of Economic Studies, vol. 66, no. 4, pp. 873-907, 1999.

[5] L. Hansen, TH. Sargent, G. A. Turluhambetova, and N. Williams, "Robustness and uncertainty aversion," Working Paper, 2002.

[6] Z. Chen and L. G. Epstein, "Ambiguity, risk, and asset returns in continuous time," Econometrica, vol. 70, no. 4, pp. 1403-1443, 2002. 
[7] L. G. Epstein and T. Wang, "Uncertainty, risk-neutral measures and security price booms and crashes," Journal of Economic Theory, vol. 67, pp. 40-80, 1995.

[8] B. Routledge and S. Zin, "Model Uncertainty and Liquidity," National Bureau of Economic Research 8683, 2001.

[9] I. Gilboa and D. Schmeidler, "Maxmin expected utility with non-unique prior," Journal of Mathematical Economics, vol. 18, no. 2, pp. 141-153, 1989.

[10] P. Artzner, F. Delbaen, J.-M. Eber, and D. Heath, "Coherent measures of risk," Mathematical Finance, vol. 9, no. 3, pp. 203228, 1999.

[11] H. Föllmer and A. Schied, "Convex measures of risk and trading constraints," Finance and Stochastics, vol. 6, no. 4, pp. 429-447, 2002.

[12] S. Peng, "Backward SDE and related g-expectation," in Backward Stochastic Differential Equations, E. N. Karoui and N. Mazliak, Eds., vol. 364 of Pitman Research Notes in Mathematics, pp. 141-159, Longman, Harlow, UK, 1997.

[13] S. Peng, "Filtration consistent nonlinear expectations and evaluations of contingent claims," Acta Mathematicae Applicatae Sinica, vol. 20, no. 2, pp. 1-24, 2004.

[14] M. Avellaneda, A. Levy, and A. Paras, "Pricing and hedging derivative securities in markets with uncertain volatilities," Applied Mathematical Finance, vol. 2, no. 2, pp. 73-88, 1995.

[15] F. Delbaen, "Coherent measures of risk on general probability space," in Advances in Finance and Stochastics, Essays in Honor of Dieter Sondermann, K. Sandmann and P. J. Schonbucher, Eds., pp. 1-37, Springer, Berlin, Germany, 2002.

[16] L. Denis and C. Martini, "A theoretical framework for the pricing of contingent claims in the presence of model uncertainty," The Annals of Applied Probability, vol. 16, no. 2, pp. 827-852, 2006.

[17] S. Peng, "Stochastic analysis and applications," in The Abel Symposium 2005, F. E. Benth, G. D. Nunno, T. Lindstrøm, B. Øksendal, and T. Zhang, Eds., pp. 541-567, 2005.

[18] S. Peng, "Law of large numbers and central limit theorem under nonlinear expectations," https://arxiv.org/abs/math/0702358, 2007.

[19] S. Peng, "Multi-dimensional G-Brownian motion and related stochastic calculus under G-expectation," Stochastic Processes and Their Applications, vol. 118, no. 12, pp. 2223-2253, 2008.

[20] Y. Fan, "The pricing of asian options in uncertain volatility model," Mathematical Problems in Engineering, vol. 2014, Article ID 786391, 16 pages, 2014.

[21] D. Duffie, Dynamic Asset Pricing Theory, Princeton University Press, Princeton, NJ, USA, 1992.

[22] S. G. Peng, "Survey on normal distributions, central limit theorem, Brownian motion and the related stochastic calculus under sublinear expectations," Science China Mathematics, vol. 52, no. 7, pp. 1391-1411, 2009.

[23] N. El Karoui and M.-C. Quenez, "Dynamic programming and pricing of contingent claims in an incomplete market," SIAM Journal on Control and Optimization, vol. 33, no. 1, pp. 29-66, 1995.

[24] N. El Karoui, S. Peng, and M. C. Quenez, "Backward stochastic differential equations in finance," Mathematical Finance, vol. 7, no. 1, pp. 1-71, 1997.

[25] S. Peng, "Nonlinear Expectations and Stochastic Calculus under Uncertainty," https://arxiv.org/abs/1002.4546, 2010.

[26] P. Wilmott, Paul Wilmott on Quantitave Finance, John Wiley, Chichester, UK, 2000.
[27] S. Chaumont, "A strong comparison result for viscosity solutions to hamilton-jacobi-bellman equations with dirichlet conditions on a non-smooth boundary," Working Paper, 2004.

[28] G. Barles, "Convergence of numerical schemes for degenerate parabolic equations arising in finance theory," in Numerical Methods in Finance, L. C. G. Rogers and D. Talay, Eds., vol. 13, pp. 1-21, Cambridge University Press, Cambridge, UK, 1997. 


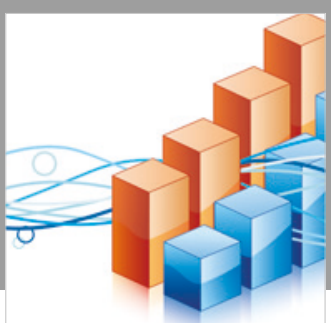

Advances in

Operations Research

\section{-n-m}
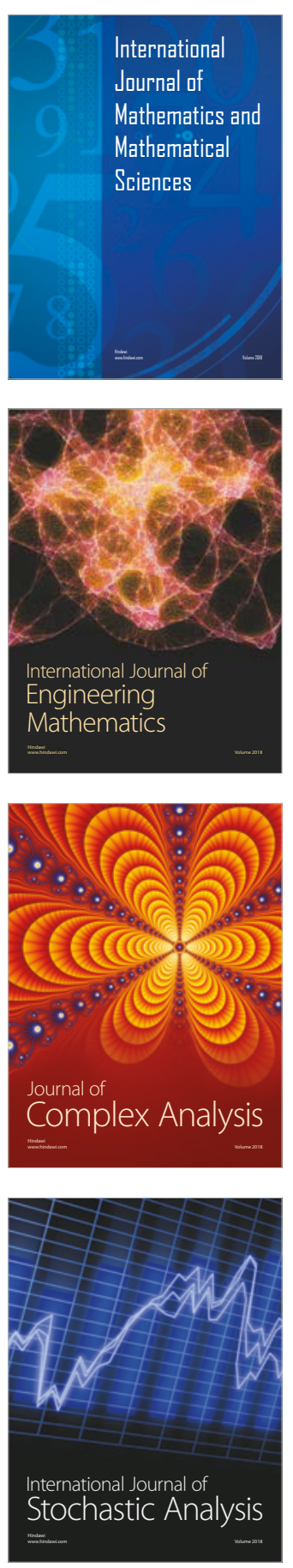
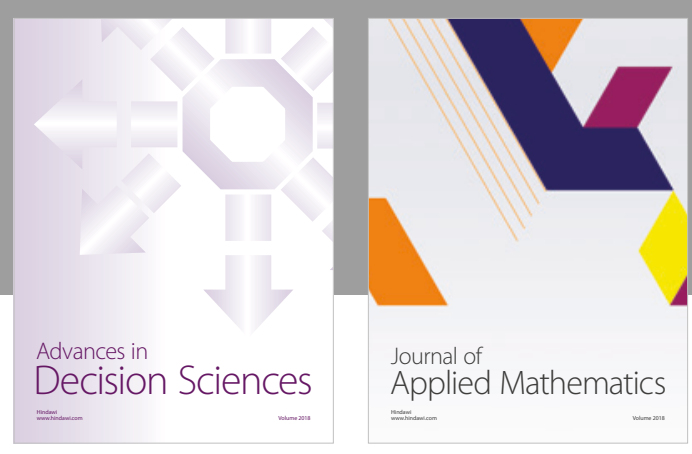

Journal of

Applied Mathematics
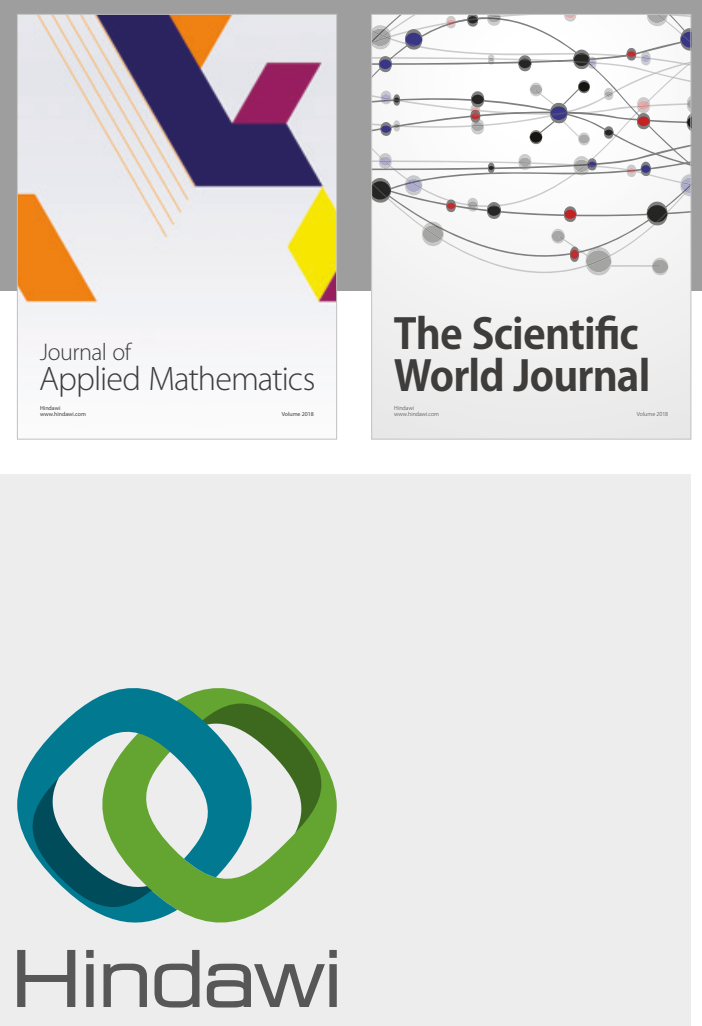

Submit your manuscripts at

www.hindawi.com

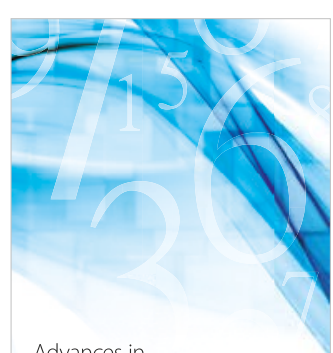

Advances in
Numerical Analysis
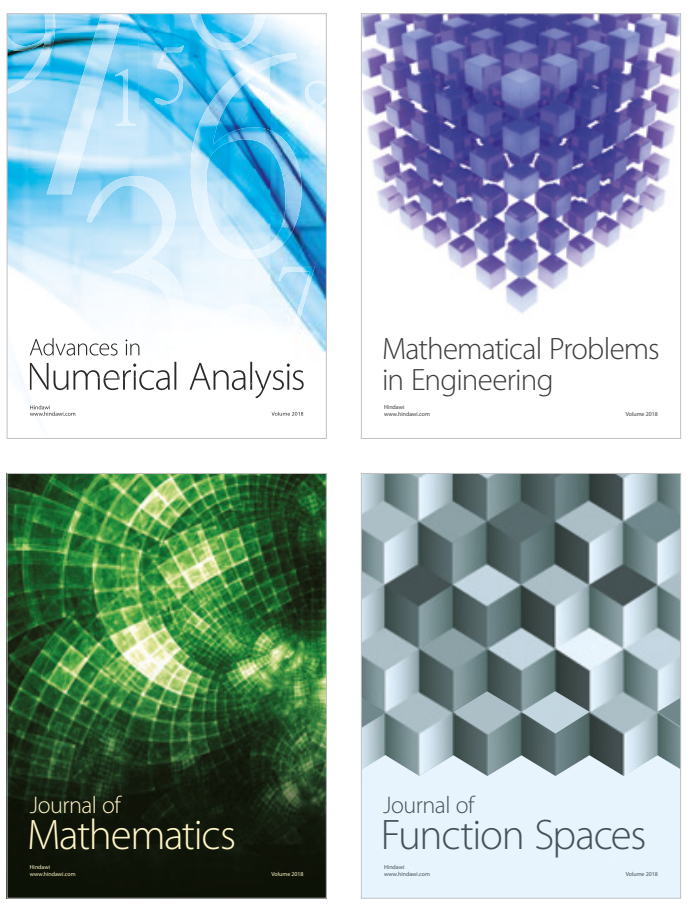

Mathematical Problems in Engineering

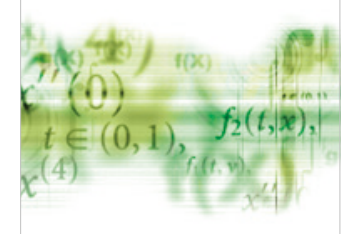

International Journal of

Differential Equations

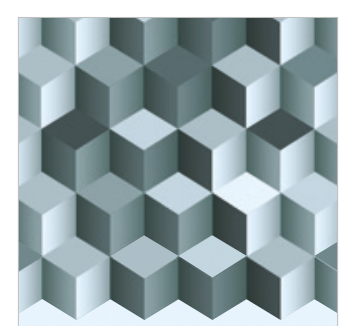

Journal of

Function Spaces

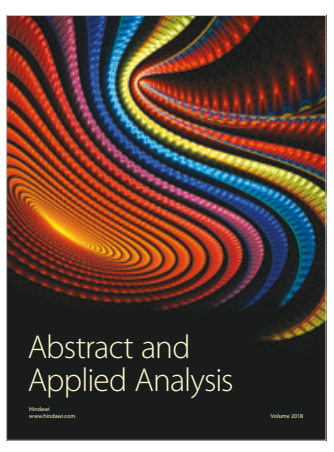

The Scientific

World Journal

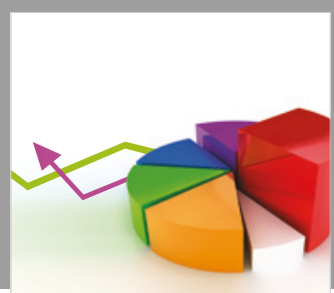

Journal of

Probability and Statistics
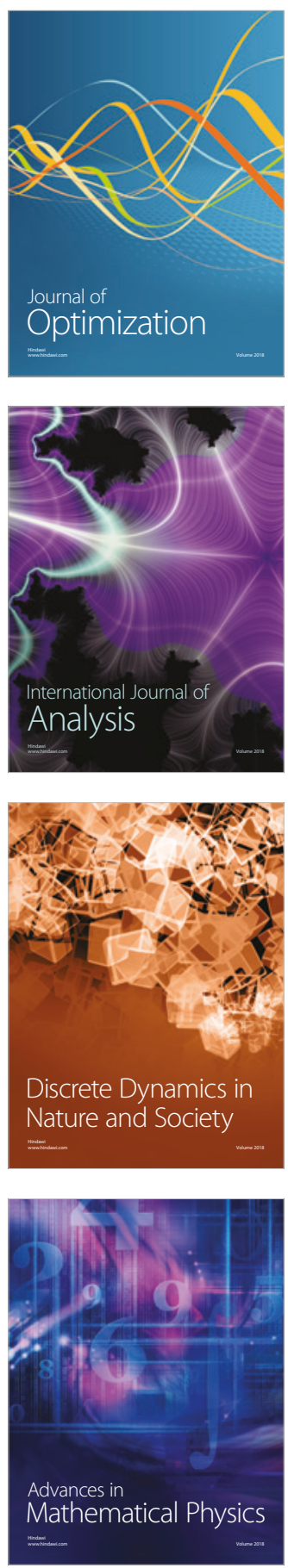